\title{
A Direct Adaptive Control Approach in the Presence of Model Mismatch
}

\author{
Suresh M. Joshi* $\quad$ Gang Tao ${ }^{\dagger} \quad$ Thuan Khong $^{\ddagger}$
}

\begin{abstract}
This paper considers the problem of direct model reference adaptive control when the plant-model matching conditions are violated due to abnormal changes in the plant or incorrect knowledge of the plant's mathematical structure. The approach consists of direct adaptation of state feedback gains for state tracking, and simultaneous estimation of the plant-model mismatch. Because of the mismatch, the plant can no longer track the state of the original reference model, but may be able to track a new reference model that still provides satisfactory performance. The reference model is updated if the estimated plant-model mismatch exceeds a bound that is determined via robust stability and/or performance criteria. The resulting controller is a hybrid direct-indirect adaptive controller that offers asymptotic state tracking in the presence of plant-model mismatch as well as parameter deviations.
\end{abstract}

\section{Introduction}

Adaptive control methodologies provide mechanisms that adjust a controller for a system under parametric, structural, and environmental uncertainties to achieve both stability and tracking. Direct model reference adaptive control (MRAC) has been known as an effective method for state or output tracking. In particular, the 'state feedback-for-state-tracking (SFST)' adaptive architecture has the advantages of relatively simple implementation and effective state tracking in the presence of parameter uncertainties as well as actuator failures $[1,2,3]$. However, to ensure stability and asymptotic state tracking, the SFST control law requires rather stringent plant-model matching conditions to be satisfied. The reference model design is usually based on the nominal model of the plant, and incorporates the desired closed-loop performance characteristics. Thus the nominal plant satisfies the matching conditions. In reality, however, the actual plant parameters differ from the nominal values, because of modeling errors, uncertainties, and parameter variation. If these differences are such that the matching conditions are still satisfied, the SFST adaptive control law can still ensure stability and state tracking. However, in many cases, the parameter changes may alter the mathematical structure of the systems (e.g., due to icing or damage in aircraft), and it may no longer be possible to meet the plant-model matching conditions. Since the proofs of stability (signal boundedness) and asymptotic tracking assume matching conditions, they are no longer valid in the presence of mismatch. Therefore plant-model mismatch has been recognized as an important problem and has been considered in the literature [4], [5].

If the changes in the plant are moderately small, it may still be possible to follow a slightly perturbed reference model that has good stability and performance characteristics. If the changes in the plant are large, it would be necessary to redesign the reference model, so that it has satisfactory performance and the matching conditions are once again met. In any case, it is important to estimate the plant-model mismatch while simultaneously performing control gain adaptation and maintaining stability and tracking. With this philosophy, this paper presents a direct adaptive control method for simultaneously estimating the plantmodel mismatch while accomplishing state tracking. The resulting approach is a hybrid direct/indirect adaptive approach wherein the reference model is updated when the estimated mismatch exceeds a predetermined threshold. This approach is first developed for the case without failures and then extended

\footnotetext{
${ }^{*}$ Senior Scientist, Mail Stop 308, NASA Langley Research Center, Hampton, VA 23681; Email suresh.m.joshi@nasa.gov; Fellow-AIAA.

${ }^{\dagger}$ Professor, Department of Electrical and Computer Engineering, University of Virginia, Charlottesville, VA, 22904; Email gt9s@virginia.edu; Member-AIAA.

${ }^{\ddagger}$ Research Engineer,Mail Stop 308, NASA Langley Research Center, Hampton, VA 23681; Email thuan.khong@nasa.gov
} 
to include actuator failures. The primary purpose of the paper is to focus on theoretical aspects and to suggest a method for systematically updating the reference model while maintaining signal boundedness and asymptotic tracking.

The paper is organized as follows. Section II develops an adaptive control scheme for the case when there are no faults, but unmatchable structural changes occur in the system- and input- matrices of the plant. Section III extends the adaptive scheme to the case when multiple actuator failures can occur in addition to unmatchable uncertainties. Simulation results are presented in Section IV to demonstrate the methods, and Sections V and VI include some discussion and concluding remarks.

\section{Adaptive Control with Model Update- No Faults}

We will focus on linear time-invariant systems (plant) described by:

$$
\dot{x}(t)=\left(A_{0}+\delta a\right) x(t)+\left(B_{0}+\delta b\right) u(t),
$$

where $A_{0}, \delta a \in R^{n \times n}, B_{0}, \delta b \in R^{n \times m}, x(t) \in R^{n}$, and $u(t) \in R^{m} . A_{0} B_{0}$ represent the nominal values of the system parameters, and $\delta a, \delta b$ represent unknown parameter deviations, which are assumed to be constant.

The control objective is to design an adaptive state feedback control signal $u(t) \in R^{m}$ to be applied to all $m$ of the actuators so that the plant's state vector tracks the state vector of a reference model given by:

$$
\dot{x}_{m}(t)=A_{m} x_{m}(t)+B_{m} r(t),
$$

where $x_{m} \in R^{n}$ is the reference model state vector, $A_{m} \in R^{n \times n}, B_{m} \in R^{n \times m}$, and $r(t) \in R^{m_{r}}\left(1 \leq m_{r} \leq m\right)$ is a bounded reference input, chosen for some desired system behavior. The objective is to design an adaptive control law that will ensure closed-loop signal boundedness and asymptotic tracking despite uncertainties, i.e., $\lim _{t \rightarrow \infty}\left(x(t)-x_{m}(t)\right)=0$.

The nominal system $\left(A_{0}, B_{0}\right)$ and the reference model are assumed to satisfy the SFST matching conditions, i.e., there exist gains $K_{1} \in R^{n \times m}, K_{2} \in R^{m \times m_{r}}$ such that

$$
A_{m}=A_{0}+B_{0} K_{1}^{T} ; B_{m}=B_{0} K_{2}
$$

The reference model is assumed to capture the desired closed-loop response of the plant. The reference model may be designed, for example, using methods such as LQR, $H_{2}$, or $H_{\infty}$, based on imperfect knowledge of the plant. The gains $K_{1}, K_{2}$ designed in this manner are used as initial estimates of the adaptive gains. For the adaptive control scheme, only $A_{m}$ and $B_{0}$ need to be known.

In applications such as aircraft flight control, because of model errors, damage, or changes such as icing, the actual plant, is $\left(A_{0}+\delta a, B_{0}+\delta b\right)$, and therefore, the matching conditions may not be satisfied for any $K_{1}, K_{2}$. When the matching conditions are violated, stability (signal boundedness) and asymptotic tracking are no longer guaranteed.

As a simple generic example, suppose

$$
\begin{gathered}
A_{0}=\left(\begin{array}{cc}
0 & 1 \\
-1 & -2
\end{array}\right) ; \quad B_{0}=\left(\begin{array}{c}
0 \\
1
\end{array}\right) ; \delta a=\left(\begin{array}{cc}
\delta a_{11} & \delta a_{12} \\
\delta a_{21} & \delta a_{22}
\end{array}\right) ; \delta b=\left(\begin{array}{c}
\delta b_{1} \\
\delta b_{2}
\end{array}\right) \\
A_{m}=\left(\begin{array}{cc}
0 & 1 \\
-3 & -5
\end{array}\right) ; \quad B_{m}=\left(\begin{array}{c}
0 \\
2
\end{array}\right)
\end{gathered}
$$

The perturbations $\delta a_{21}, \delta a_{22}$, and $\delta b_{2}$ can be compensated perfectly (matched) by some state feedback gains $K_{1}, K_{2}$. However, structural perturbations $\delta a_{11}, \delta a_{12}$, and $\delta b_{1}$ cannot be matched by state feedback control law. Thus we can take $\delta a$ and $\delta b$ to be the "unmatched" uncertainties,

$$
\delta a=\left(\begin{array}{cc}
\delta a_{11} & \delta a_{12} \\
0 & 0
\end{array}\right) ; \quad \delta b=\left(\begin{array}{c}
\delta b_{1} \\
0
\end{array}\right)
$$

Returning to the general problem, define the control law as:

$$
u=\widehat{K}_{1}^{T} x+\widehat{K}_{2} r
$$


where $\widehat{K}_{1}, \widehat{K}_{2}$ denote the (time-varying) estimates of $K_{1}, K_{2}$.

The closed-loop plant is given by:

$$
\begin{aligned}
\dot{x} & =\left(A_{0}+\delta a\right) x+\left(B_{0}+\delta b\right)\left(\widehat{K}_{1}^{T} x+\widehat{K}_{2} r\right) \\
& =\left(A_{0}+\delta a\right) x+B_{0}\left(K_{1}^{T} x+K_{2} r\right)+B_{0}\left(\widetilde{K}_{1}^{T} x+\widetilde{K}_{2} r\right)+\delta b\left(\widehat{K}_{1}^{T} x+\widehat{K}_{2} r\right) \\
& =\left(A_{0}+B_{0} K_{1}^{T}+\delta a\right) x+B_{0} K_{2} r+B_{0}\left(\widetilde{K}_{1}^{T} x+\widetilde{K}_{2} r\right)+\delta b\left(\widehat{K}_{1}^{T} x+\widehat{K}_{2} r\right) \\
& =\left(A_{m}+\delta a+\delta b \widehat{K}_{1}^{T}\right) x+\left(B_{m}+\delta b \widehat{K}_{2}\right) r+B_{0}\left(\widetilde{K}_{1}^{T} x+\widetilde{K}_{2} r\right)
\end{aligned}
$$

where $\widetilde{K}_{1}=\widehat{K}_{1}-K_{1} ; \widetilde{K}_{2}=\widehat{K}_{2}-K_{2}$.

To accommodate plant-model mismatch and parameter deviation, modify the reference model as:

$$
\dot{x}_{m}=\left(A_{m}+\widehat{\delta a}+\widehat{\delta b} \widehat{K}_{1}^{T}\right) x_{m}+\left(B_{m}+\widehat{\delta b} \widehat{K}_{2}\right) r
$$

where $\widehat{\delta a}, \widehat{\delta b}$ denote (time-varying) estimates of $\delta a$ and $\delta b$.

Denote

$$
\widehat{\Delta A}=\widehat{\delta a}+\widehat{\delta b} \widehat{K}_{1}^{T} ; \widehat{\Delta B}=\widehat{\delta} b \widehat{K}_{2}
$$

so that

$$
A_{M}=A_{m}+\widehat{\Delta A} ; B_{M}=B_{m}+\widehat{\Delta B}
$$

which are time-varying matrices. The modified time-varying reference model is

$$
\dot{x}_{m}=A_{M} x_{m}+B_{M} r
$$

This representation of the reference model indicates that the plant can no longer follow the original reference model (2), but can possibly follow the perturbed reference model (12). Therefore, it will be necessary to first ensure that the perturbed reference model has acceptable stability and performance characteristics. Use of a time-varying reference model, which is a departure from conventional MRAC, was addressed in [7], which employed a time-varying Lyapunov function weighting matrix $(P(t))$. In the present approach, a time-invariant Lyapunov function weighting matrix is used along with a quadratic stability argument to ensure stability of the time-varying reference model. This can be accomplished by calculating the permissible limits on the estimated perturbations such that the stability and performance will remain satisfactory for all (time-varying) perturbations within these limits. Subsequently it will also be necessary to derive adaptive laws (for updating $\widehat{K_{1}}, \widehat{K_{2}}, \widehat{\delta a}, \widehat{\delta b}$ ) that guarantee that $x(t) \rightarrow x_{m}(t)$, and that all signals remain bounded.

\section{A. Stability and Performance of Perturbed Reference Model}

$A_{M}$ is a time-varying matrix which can be expressed as an affine function of a parameter vector $\mathbf{p} \in R^{n_{p}}$ that lies in a convex polytope $\mathcal{S}$ having vertices $\mathbf{p}^{\mathbf{j}}, j=1, \ldots n_{v}$. For example, $A_{M}$ can be expressed as

$$
A_{M}(\mathbf{p}(t))=A_{m}+\sum_{1}^{n_{p}} \mathbf{p}_{\mathbf{i}}(t) A_{M_{i}}
$$

where $A_{M_{i}}$ are constant matrices and $\mathbf{p}_{\mathbf{i}}(t) \in\left[\underline{\mathbf{p}_{\mathbf{i}}}, \overline{\mathbf{p}_{\mathbf{i}}}\right],\left(\mathbf{p}_{\mathbf{i}}\right.$ is the $i$ th component of $\left.\mathbf{p}\right)$. In this case $\mathcal{S}$ is a hyper-rectangular region with $n_{v}=2^{n_{p}}$ vertices. Let $A_{M}\left(\mathbf{p}^{\mathbf{j}}\right)$ denote the value of the reference model system matrix at vertex $\mathbf{p}^{\mathbf{j}}$. Suppose there exist positive definite matrices $P=P^{T}, Q=Q^{T} \in R^{n \times n}$, such that

$$
A_{M}\left(\mathbf{p}^{\mathbf{j}}\right)^{T} P+P A_{M}\left(\mathbf{p}^{\mathbf{j}}\right)<-Q, j=1, \ldots n_{v}
$$

For a given $Q,(14)$ represents a set of $n_{v}$ linear matrix inequalities (LMIs) in the unknown variable $P$. In view of (14), the Lyapunov inequality

$$
A_{M}(\mathbf{p}(t))^{T} P+P A_{M}(\mathbf{p}(t))<-Q, j=1, \ldots n_{v}
$$

is satisfied $\forall \mathbf{p}(t) \in \mathcal{S}$, and the autonomous part of the reference model (i.e., Eq. 12 with $r=0$ ) is exponentially stable $\forall \mathbf{p}(t) \in \mathcal{S}$ with a guaranteed minimum decay rate $e^{-\lambda_{\min }\left(P^{-1} Q\right) t}$. 
Express $\underline{\mathbf{p}_{\mathbf{i}}}$ and $p_{i}$ as

$$
\underline{\mathbf{p}_{\mathbf{i}}}=\theta \underline{\delta_{i}} ; \quad \overline{\mathbf{p}_{\mathbf{i}}}=\theta \overline{\delta_{i}} ; \quad i=1, \ldots n_{p}
$$

where $\delta_{i}, \overline{\delta_{i}}$ are known constants, and $\delta_{i} \leq 0 \leq \overline{\delta_{i}}$. The problem is to obtain the maximal region $\mathcal{S}$ for which (14) holds, i.e., find a positive definite symmetric matrix $P$ that maximizes $\theta$ subject to the set of LMIs in (14).

The estimate of $\mathcal{S}$ obtained in this manner is usually conservative. If the parameter variations $(\dot{p})$ are rate-bounded, less conservative estimates can be obtained. The stability of the reference model depends only on $\widehat{\delta A}$, while $\widehat{\delta B}$ affects the closed-loop gain; therefore, if $\widehat{\delta B}$ is reasonably small, the performance of the modified reference model would be acceptable.

Alternatively, instead of just quadratic stability, an $\mathrm{H}_{2}$ or $H_{\infty}$ performance requirement can be imposed on the reference model dynamics, $A_{M}, B_{M}$. Such requirements can be formulated as linear matrix inequalities at the vertices of a polytope in the parameter space and an estimate of the maximal region $\mathcal{S}$ can be obtained, thus providing the permissible bounds on the elements of $\widehat{\Delta A}$. The reference model is updated if its parameters approach the polytope boundaries.

\section{B. Adaptation Laws}

Denote $e=x-x_{m}$, Eqs. (8) and (9) yield

$$
\dot{e}=A_{M} e-\widetilde{\delta a x}-\widetilde{\delta b} u+B_{0}\left(\widetilde{K}_{1}^{T} x+\widetilde{K}_{2} r\right)
$$

For simplicity of presentation, we shall first assume that $\widehat{\Delta A}$ remains within the quadratic stability bounds. Subsequently in Section IIC we shall present a parameter projection algorithm to ensure that this condition is met. We have the following result.

Theorem 1 For the system given by (1), (9), the adaptive controller (7) with gain adaptation laws

$$
\begin{aligned}
& \dot{\widehat{K_{1}}}=-\Gamma_{1} x e^{T} P B_{0} \\
& \dot{\widehat{K_{2}}}=-\Gamma_{2} B_{0}^{T} P e r^{T}
\end{aligned}
$$

and parameter estimation laws

$$
\begin{aligned}
\dot{\hat{\delta a}} & =\Gamma_{a} P e x^{T} \\
\dot{\hat{\delta_{b}}} & =\Gamma_{b} P e u^{T}
\end{aligned}
$$

where $\Gamma_{1}, \Gamma_{2}, \Gamma_{a}, \Gamma_{b}$ are symmetric positive definite matrices, guarantee that all closed-loop signals including adaptive gains and parameter estimates are bounded and the tracking error $e(t)=x(t)-x_{m}(t)$ goes to zero as $t \rightarrow \infty$.

Outline of Proof- Define

$$
V=e^{T} P e+\sum_{1}^{n}{\widetilde{\delta a_{i}}}^{T} \Gamma_{a}^{-1} \widetilde{\delta a_{i}}+\sum_{1}^{m}{\widetilde{\delta b_{i}}}^{T} \Gamma_{b}^{-1} \widetilde{\delta b_{i}}+\sum_{1}^{m}{\widetilde{K_{1 i}}}^{T} \Gamma_{1}^{-1} \widetilde{K_{1} i}+\sum_{1}^{m_{r}}{\widetilde{K_{2 i}}}^{T} \Gamma_{2}^{-1} \widetilde{K_{2 i}}
$$

where the subscript $i$ denotes the $i^{\text {th }}$ column of $\widetilde{\delta a}, \widetilde{\delta b}, \widetilde{K_{1}}$, and $\widetilde{K_{2}} ; \Gamma_{a} \in R^{n \times n}, \Gamma_{b} \in R^{n \times n}, \Gamma_{1} \in R^{n \times n}, \Gamma_{2} \in$ $R^{m \times m}$ are positive definite and symmetric. Differentiating (19) with respect to $t$ and using (16), (14) as well as properties of matrix trace, the following is obtained after simplification:

$$
\begin{aligned}
\dot{V}= & e^{T} P\left[A_{M}-\widetilde{\delta a}-\widetilde{\delta b} u+B_{0}\left(\widetilde{K_{1}^{T}} x+\widetilde{K_{2}} r\right)\right] \\
& +2 \operatorname{Tr}\left[\widetilde{\delta a}^{T} \Gamma_{a}^{-1} \dot{\hat{\delta a}}\right]+2 \operatorname{Tr}\left[\widetilde{\delta b}^{T} \Gamma_{b}^{-1} \dot{\hat{\delta} b}\right] \\
& +2 \operatorname{Tr}\left[{\widetilde{K_{1}}}^{T} \Gamma_{1}^{-1} \dot{\hat{K_{1}}}\right]+2 \operatorname{Tr}\left[{\widetilde{K_{2}}}^{T} \Gamma_{2}^{-1} \dot{\hat{K}_{2}}\right]
\end{aligned}
$$

In writing the above equation, we have used the fact that $\dot{\widetilde{K_{1}}}=\dot{\widehat{K_{1}}}$ and the property:

$$
\operatorname{Tr}\left[X R X^{T}\right]=\sum_{1}^{m} x_{i}^{T} R x_{i}
$$


where $X \in R^{m \times n}, R \in R^{n \times n}$ and $x_{i}^{T}$ denotes the $i$ th row of $X$. We also have:

$$
\begin{aligned}
e^{T} P \widetilde{\delta a x} & =x^{T} \widetilde{\delta a}^{T} P e=\operatorname{Tr}\left[\widetilde{\delta a}^{T} P e x^{T}\right] \\
e^{T} P \widetilde{\delta b} u & =u^{T} \widetilde{\delta b}^{T} P e=\operatorname{Tr}\left[\widetilde{\delta b}^{T} P e u^{T}\right] \\
e^{T} P B_{0} \widetilde{K}_{1}^{T} x & =\operatorname{Tr}\left[\widetilde{K}_{1}^{T} x e^{T} P B_{0}\right] \\
e^{T} P B_{0} \widetilde{K}_{2} r & =\operatorname{Tr}\left[r^{T} \widetilde{K}_{2}^{T} B_{0}^{T} P e\right]=\operatorname{Tr}\left[\widetilde{K}_{2}^{T} B_{0}^{T} P e r^{T}\right]
\end{aligned}
$$

Therefore,

$$
\begin{aligned}
\dot{V} \leq & -e^{T} Q e-2 \operatorname{Tr}\left[\widetilde{\delta a}^{T}\left\{P e x^{T}-\Gamma_{a}^{-1} \dot{\hat{\delta} a}\right\}\right]-2 \operatorname{Tr}\left[\widetilde{\delta b}^{T}\left\{P e u^{T}-\Gamma_{b}^{-1} \dot{\hat{\delta} b}\right\}\right] \\
& +2 \operatorname{Tr}\left[\widetilde{K}_{1}^{T}\left\{x e^{T} P B_{0}+\Gamma_{1}^{-1} \dot{\widehat{K}}_{1}^{T}\right\}+2 \operatorname{Tr}\left[\widetilde{K}_{2}^{T}\left\{B_{0}^{T} P e r^{T}+\Gamma_{2}^{-1} \dot{\widehat{K}}_{2}\right\}\right]\right.
\end{aligned}
$$

The update laws for $\widehat{K_{1}} \widehat{K_{2}}, \widehat{\delta a}$, and $\widehat{\delta b}$, in (17), (18) were chosen to make all terms except the first term in the RHS of (22) equal to zero, and

$$
\dot{V} \leq-e^{T} Q e
$$

That is, $V(T)$ is bounded for all $T$, and $e(t), x(t), \widehat{K_{1}}, \widehat{K 2}, \widehat{\delta a}, \widehat{\delta b}$ are all bounded and $e(t) \in L^{2}$. From (16) and closed-loop signal boundedness, we have $\dot{e} \in L^{\infty}$, therefore $\lim _{t \rightarrow \infty} e(t)=0$. Because $e(t) \in L^{2} \cap L^{\infty}$ and $x(t) \in L^{\infty}, \dot{\overline{K_{1 j}}}, \dot{\overline{K_{2 j}}}, \dot{\hat{\delta a}}, \dot{\hat{\delta b}} \in L^{2} \cap L^{\infty}$. It can be verified that $\ddot{\overline{K_{1 j}}}, \ddot{\ddot{K_{2 j}}}, \ddot{\hat{\delta a}}, \ddot{\hat{\delta b}} \in L^{\infty}$, therefore $\lim _{t \rightarrow \infty} \widehat{K_{1 j}(t)}=0 ; \quad \lim _{t \rightarrow \infty} \widehat{K_{2 j}(t)}=0, \quad \lim _{t \rightarrow \infty} \hat{\delta a(t)}=0 ; \quad \lim _{t \rightarrow \infty} \widehat{\dot{\delta b(t)}}=0$. That is, all signals and estimates are bounded, and $\lim _{t \rightarrow \infty}\left(x(t)-x_{m}(t)\right)=0$.

\section{Remarks}

- It would be necessary to use projection methods (e.g., $[2,6])$ to ensure that the parameter estimates remain within the polytope. The reference model is updated when the parameter estimates approach the polytope boundaries. The parameter projection is addressed in the next subsection.

- For the case where only some of the elements of $A$ and $B$ are unmatched, the parameter estimation laws can be simplified. For the generic example in (4), the parameters updates laws can be written as

$$
\begin{aligned}
& \dot{\widehat{\delta a}}_{11}=\varepsilon_{1}^{T} \Gamma_{a} \operatorname{Pex}^{T} \varepsilon_{1} \\
& \dot{\widehat{\delta a}}_{12}=\varepsilon_{1}^{T} \Gamma_{a} P e x^{T} \varepsilon_{2}
\end{aligned}
$$

where $\varepsilon_{i} \in R^{n}$ denotes a unit vector whose $i$ th element is unity and all other elements are zero. In fact, the update laws in Theorem 1 can be easily modified for individual $\widehat{\delta a_{i j}}$ 's, with individual gains $\Gamma_{i j}$.

- If sufficient persistent excitation is present, $\widetilde{\delta} a, \widetilde{\delta} b$ should converge to zero.

\section{Parameter Projection}

The stability condition in (14) requires that the reference model system matrix $A_{M}(t)=A_{m}+\widehat{\delta a}+\widehat{\delta b} \widehat{K}_{1}^{T}=$ $A_{m}+\widehat{\Delta A}$ be a stable matrix for all possible parameter estimates $\widehat{\delta a}, \widehat{\delta b}$ and $\widehat{K}_{1}$. From subsection A, the stability of $A_{M}$ is assured if $\widehat{\Delta A}$ remains within the bounds obtained from maximizing $\theta$ in (15), denoted as $\left({\widehat{\Delta A_{i j}}}^{a},{\widehat{\Delta A_{i j}}}^{b}\right)$. The adaptive laws (17)-(18) generating these parameter estimates do not automatically guarantee such a property, and they need to be re-designed with parameter projection.

To implement a parameter projection adaptive law, we need the knowledge of the ranges of the nominal parameters to be estimated. In this case, we need to know the lower and upper bounds $\delta a_{i j}^{a}$ and $\delta a_{i j}^{b}$ of the components $\delta a_{i j}$ of $\delta a=\left\{\delta a_{i j}\right\} \in R^{n \times n}, \delta b_{i j}^{a}$ and $\delta b_{i j}^{b}$ of the components $\delta b_{i j}$ of $\delta b=\left\{\delta b_{i j}\right\} \in$ $R^{n \times m}$, and $k_{1 i j}^{a}$ and $k_{1 i j}^{b}$ of the components $k_{1 i j}$ of $K_{1}=\left\{k_{1 i j}\right\} \in R^{n \times n}$, such that for any $\widehat{\delta a}$ whose 
components $\widehat{\delta a}_{i j} \in\left[\delta a_{i j}^{a}, \delta a_{i j}^{b}\right]$, any $\widehat{\delta b}$ whose components $\widehat{\delta b}_{i j} \in\left[\delta b_{i j}^{a}, \delta b_{i j}^{b}\right]$, and any $\widehat{K}_{1}$ whose components $\widehat{k}_{1 i j} \in\left[k_{1 i j}^{a}, k_{1 i j}^{b}\right]$, the matrix $A_{M}=A_{m}+\widehat{\delta a}+\widehat{\delta b} \widehat{K}_{1}^{T}$ is stable. Such lower and upper bounds are to be used in the construction of the parameter projection based adaptive laws. This implies that the parameter uncertainties $\delta a$ and $\delta b$ should stay within the parameter bounds after the adaptive laws are constructed with such bounds, in order to ensure the stability of the adaptive laws. Since $\widehat{\Delta A}$ depends on $\widehat{\delta a}, \widehat{\delta b}$, and $\widehat{K_{1}}$, it would be necessary to determine the bounds $\left[\delta a_{i j}^{a}, \delta a_{i j}^{b}\right],\left[\delta b_{i j}^{a}, \delta b_{i j}^{b}\right],\left[k_{1 i j}^{a}, k_{1 i j}^{b}\right]$, such that $\widehat{\Delta A}$ remains within the bounds $\left[{\widehat{\Delta A_{i j}}}^{a},{\widehat{\Delta A_{i j}}}^{b}\right]$. Investigating appropriate methods for this mapping remains an area of future research.

There are two expected desirable properties of parameter projection: one is to ensure $\widehat{\delta a}_{i j} \in\left[\delta a_{i j}^{a}, \delta a_{i j}^{b}\right]$, $\widehat{\delta b}_{i j} \in\left[\delta b_{i j}^{a}, \delta b_{i j}^{b}\right]$, and $\widehat{k}_{1 i j} \in\left[k_{1 i j}^{a}, k_{1 i j}^{b}\right]$ for all $t \geq 0$ so that $A_{M}$ is stable, and the other is to maintain the desired Lyapunov stability properties established for the case without parameter projection. For this purpose, the adaptive laws (17)-(18) are modified as

$$
\begin{aligned}
\dot{\hat{K_{1}}} & =-\Gamma_{1} x e^{T} P B_{0}+F_{1} \\
\dot{\widehat{K_{2}}} & =-\Gamma_{2} B_{0}^{T} P e r^{T} \\
\dot{\hat{\delta a}} & =\Gamma_{a} P e x^{T}+F_{a} \\
\dot{\hat{\delta_{b}}} & =\Gamma_{b} P e u^{T}+F_{b}
\end{aligned}
$$

where $F_{1}(t)=\left\{f_{1 i j}\right\} \in R^{n \times m}, F_{a}(t)=\left\{f_{a i j}\right\} \in R^{n \times n}$ and $F_{b}(t)=\left\{f_{b i j}\right\} \in R^{n \times m}$ are projection functions with their $i j$ th components being $f_{1 i j}, f_{a i j}$ and $f_{b i j}$, respectively, and the corresponding adaptation gain matrices are chosen to be $\Gamma_{1}=\operatorname{diag}\left\{\gamma_{11}, \gamma_{12}, \ldots, \gamma_{1 n}\right\}, \Gamma_{a}=\operatorname{diag}\left\{\gamma_{a 1}, \gamma_{a 2}, \ldots, \gamma_{a n}\right\}$ and $\Gamma_{b}=$ $\operatorname{diag}\left\{\gamma_{b 1}, \gamma_{b 2}, \ldots, \gamma_{b n}\right\}$, with $\gamma_{1 i}>0, \gamma_{a i}>0$ and $\gamma_{b i}>0$, for $i=1,2, \ldots, n$, while $\Gamma_{2}=\Gamma_{2}^{T}>0$ with $\Gamma_{2} \in R^{m \times m}$, which can be non-diagonal, as parameter projection is not needed for $\widehat{K_{2}}$.

To specify the projection functions $F_{1}(t), F_{a}(t)$ and $F_{b}(t)$, we denote

$$
\begin{aligned}
G_{1} & =-\Gamma_{1} x e^{T} P B_{0} \\
G_{a} & =\Gamma_{a} P e x^{T} \\
G_{b} & =\Gamma_{b} P e u^{T}
\end{aligned}
$$

with their $i j$ th components being $g_{1 i j}, g_{a i j}$ and $g_{b i j}$, respectively, that is, $G_{1}(t)=\left\{g_{1 i j}\right\} \in R^{n \times m}, G_{a}(t)=$ $\left\{g_{a i j}\right\} \in R^{n \times n}$ and $G_{b}(t)=\left\{g_{b i j}\right\} \in R^{n \times m}$, and similarly, $\widehat{\delta a}=\left\{\widehat{\delta a}_{i j}\right\}, \widehat{\delta b}=\left\{\widehat{\delta b}_{i j}\right\}$ and $\widehat{K}_{1}=\left\{\widehat{k}_{1 i j}\right\}$.

We then choose the initial parameter estimates to satisy

$$
\widehat{\delta a}_{i j}(0) \in\left[\delta a_{i j}^{a}, \delta a_{i j}^{b}\right], \widehat{\delta b}_{i j}(0) \in\left[\delta b_{i j}^{a}, \delta b_{i j}^{b}\right], \widehat{k}_{1 i j}(0) \in\left[k_{1 i j}^{a}, k_{1 i j}^{b}\right]
$$

and set the projection function components as

$$
\begin{aligned}
& f_{1 i j}(t)= \begin{cases}0 & \text { if } \widehat{k}_{1 i j}(t) \in\left(k_{1 i j}^{a}, k_{1 i j}^{b}\right), \text { or } \\
& \text { if } \widehat{k}_{1 i j}(t)=k_{1 i j}^{a}, g_{1 i j}(t) \geq 0, \text { or } \\
& \text { if } \widehat{k}_{1 i j}(t)=k_{1 i j}^{b}, g_{1 i j}(t) \leq 0, \\
-g_{1 i j}(t) & \text { otherwise }\end{cases} \\
& f_{a i j}(t)= \begin{cases}0 & \text { if } \widehat{\delta a}_{i j}(t) \in\left(\delta a_{i j}^{a}, \delta a_{i j}^{b}\right), \text { or } \\
& \text { if } \widehat{\delta a}_{i j}(t)=\delta a_{i j}^{a}, g_{a i j}(t) \geq 0, \text { or } \\
& \text { if } \widehat{\delta a}_{i j}(t)=\delta a_{i j}^{b}, g_{a i j}(t) \leq 0, \\
-g_{a i j}(t) & \text { otherwise }\end{cases} \\
& f_{b i j}(t)= \begin{cases}0 & \text { if } \widehat{\delta b}_{i j}(t) \in\left(\delta b_{i j}^{a}, \delta b_{i j}^{b}\right), \text { or } \\
& \text { if } \widehat{\delta b}_{i j}(t)=\delta b_{i j}^{a}, g_{b i j}(t) \geq 0, \text { or } \\
& \text { if } \widehat{\delta b}_{i j}(t)=\delta b_{i j}^{b}, g_{b i j}(t) \leq 0, \\
-g_{b i j}(t) & \text { otherwise }\end{cases}
\end{aligned}
$$

for the projection functions $F_{1}(t), F_{a}(t)$ and $F_{b}(t)$ in the adaptive laws (25)-(26). 
This choice of $F_{1}(t)=\left\{f_{1 i j}\right\}, F_{a}(t)=\left\{f_{a i j}\right\}$ and $F_{b}(t)=\left\{f_{b i j}\right\}$ ensures that $\widehat{\delta a}_{i j} \in\left[\delta a_{i j}^{a}, \delta a_{i j}^{b}\right]$, $\widehat{\delta b}_{i j} \in\left[\delta b_{i j}^{a}, \delta b_{i j}^{b}\right]$, and $\widehat{k}_{1 i j} \in\left[k_{1 i j}^{a}, k_{1 i j}^{b}\right]$ for all $t \geq 0$ so that $A_{M}$ is stable. It is verified below that the choice of $F_{1}(t), F_{a}(t)$ and $F_{b}(t)$ (whose $k$ th columns are denoted as $f_{1 k}, f_{a k}$ and $f_{b k}$, respectively) also guarantees that

$$
{\widetilde{\delta a_{k}}}^{T} \Gamma_{a}^{-1} f_{a k} \leq 0,{\widetilde{\delta b_{k}}}^{T} \Gamma_{b}^{-1} f_{b k} \leq 0,{\widetilde{K_{1 k}}}^{T} \Gamma_{1}^{-1} f_{1 k} \leq 0,
$$

where $\widetilde{\delta a_{k}}, \widetilde{\delta b_{k}}$ and $\widetilde{K_{1 k}}$ are the $k$ th colunm of $\widetilde{\delta a}=\widehat{\delta a}-\delta a, \widetilde{\delta b}=\widehat{\delta b}-\delta b$ and $\widetilde{K_{1}}=\widehat{K_{1}}-K_{1}$, respectively. To see this desired parameter projection property, we write

$$
{\widetilde{\delta a_{k}}}^{T} \Gamma_{a}^{-1} f_{a k}=\sum_{i=1}^{n} \widetilde{\delta a_{i k}} \gamma_{a i}^{-1} f_{a i k}
$$

where $\widetilde{\delta a_{i k}}$ is the $i$ th component of $\widetilde{\delta a_{k}}$. When $f_{a i k}=-g_{a i k}$, we have either $\widehat{\delta a}_{i k}(t)=a_{i k}^{a}$ and $g_{a i k}(t)<0$, or $\widehat{\delta a}_{i k}(t)=a_{i k}^{b}$ and $g_{a i k}(t)>0$, and for both cases, it follows that

$$
\widetilde{\delta a_{i k}} \gamma_{a i}^{-1} f_{a i k}=-\gamma_{a i}^{-1}\left(\widehat{\delta a}_{i k}-\delta a_{i k}\right) g_{a i k} \leq 0
$$

because $\delta a_{i k} \in\left[\delta a_{i k}^{a}, \delta a_{i k}^{b}\right]$ is the specified parameter range for $\delta a_{i k}$. Hence, we have: ${\widetilde{\delta a_{k}}}^{T} \Gamma_{a}^{-1} f_{a k} \leq 0$, and similarly, ${\widetilde{\delta b_{k}}}^{T} \Gamma_{b}^{-1} f_{b k} \leq 0$ and $\widetilde{K_{1 k}}{ }^{T} \Gamma_{1}^{-1} f_{1 k} \leq 0$.

With the parameter projection functions $F_{1}(t), F_{a}(t)$ and $F_{b}(t)$, the adaptive laws (25)-(26) introduce the terms $\sum_{i=1}^{n}{\widetilde{\delta a_{i}}}^{T} \Gamma_{a}^{-1} f_{a i}, \sum_{i=1}^{n}{\widetilde{\delta b_{i}}}^{T} \Gamma_{b}^{-1} f_{b i}$ and $\sum_{i=1}^{n}{\widetilde{K_{1 i}}}^{T} \Gamma_{1}^{-1} f_{1 i}$ (which are all non-positive as just shown) in the time-derivative $\dot{V}$ in (22) of the positive definite function $V$ defined in (19), that is, we end up with

$$
\dot{V} \leq-e^{T} Q e,
$$

so that the desired stability properties of the closed-loop system remain unchanged by parameter projection which ensures that $\widehat{\delta a}_{i j} \in\left[\delta a_{i j}^{a}, \delta a_{i j}^{b}\right], \widehat{\delta b}_{i j} \in\left[\delta b_{i j}^{a}, \delta b_{i j}^{b}\right]$, and $\widehat{k}_{1 i j} \in\left[k_{1 i j}^{a}, k_{1 i j}^{b}\right]$, to make $A_{M}(t)=A_{m}+\widehat{\delta a}+$ $\widehat{\delta b} \widehat{K}_{1}^{T}$ stable as ensured by (14).

\section{Reference Model Updates}

The results in the previous subsections suggest an approach wherein the reference model is updated when $\widehat{\Delta A}, \widehat{\Delta B}$ exceed a predetermined threshold. This is expected to occur occasionally as discrete events. Assuming that reference model updates occur finite number of times, the adaptive control scheme can be implemented as follows

1. Design a reference model $A_{m}, B_{m}$ based on the initial approximate knowledge of $A_{0}, B_{0}$ (denoted by $\left.\widehat{A_{0}}, \widehat{B_{0}}\right)$, which satisfy the matching conditions (3)

2. Compute permissible limits on the elements of $\widehat{\Delta A}, \widehat{\Delta B}$ using quadratic stability or performance criteria

3. Apply adaptive control and model update laws (7), (17), (18)

4. If any elements of $\widehat{\Delta A}, \widehat{\Delta B}$ approach the limits obtained in step (2) (e.g., within 80 per cent), replace the system parameter estimates, $\widehat{A_{0}}$ by $\widehat{A_{0}}+\widehat{\delta a}$, and $\widehat{B_{0}}$ by $\widehat{B_{0}}+\widehat{\delta b}$ (both constant) and redesign the reference model

5. Set $\widehat{\delta_{a}}=0, \widehat{\delta_{b}}=0$ and go to Step 2

\section{Simultaneous Model Mismatch and Actuator Failures}

In addition to model mismatch due to reasons such as modeling errors, icing, and damage, the actuators (e.g., control surfaces in aircraft flight control) may fail during the operation. The actuator failures are modeled in this paper as

$$
u_{j}(t)=\bar{u}_{j}, t \geq t_{j}, j \in\left\{j_{1}, j_{2}, \ldots, j_{p}\right\} \subseteq\{1,2, \ldots, m\}
$$


where the failure pattern $\left\{j_{1}, j_{2}, \ldots, j_{p}\right\}$, the failure value $\bar{u}_{j}$ (assumed to be constant), and the failure time of occurrence $t_{j}$ are all unknown. For example, an aircraft control surface may be locked at some unknown fixed value due to hydraulics failure. Let $v(t)=\left[v_{1}, v_{2}, \ldots, v_{m}\right]^{T} \in R^{m}$ be the applied (commanded) control input vector. In the presence of actuator failures, the actual input vector $u$ to the system can be described as

$$
u(t)=v(t)+\sigma(\bar{u}-v(t))=(I-\sigma) v(t)+\sigma \bar{u}
$$

where

$$
\begin{aligned}
\bar{u} & =\left[\bar{u}_{1}, \bar{u}_{2}, \ldots, \bar{u}_{m}\right]^{T} \\
\sigma & =\operatorname{diag}\left\{\sigma_{1}, \sigma_{2}, \ldots, \sigma_{m}\right\} \\
\sigma_{i} & =1 \text { if the ith actuator fails, i.e., } u_{i}=\bar{u}_{i} \\
\sigma_{i} & =0 \text { otherwise }
\end{aligned}
$$

That is, $\sigma$ is a diagonal matrix whose entries are piecewise step- or zero- functions of time. The components of the applied input signal $v(t)=\left[v_{1}(t), v_{2}(t), \ldots, v_{m}(t)\right]^{T}$, which correspond to the failed actuators, cannot affect the system dynamics. The actuator failures are uncertain in value, pattern and time of occurrence. The objective of the adaptive controller is to synthesize the control signal $v(t)$ so as to ensure the system stability and asymptotic tracking regardless of whether (or which) actuators have failed, or the failure values. That is, $v(t)$ should be capable of compensating for the failures automatically.

Direct adaptive control with actuator failures was extensively studied (e.g., [1], [2], wherein different adaptive schemes were developed, that included state feedback for state tracking (SFST), state feedback for output tracking (SFOT) and output feedback for output tracking (OFOT). While all these adaptive schemes require some assumptions in the form of plant-model matching conditions, the SFST scheme requires the most stringent matching conditions. However, the SFST scheme has the advantage of simplicity, and is well-suited in aircraft flight control applications because the state vector measurements are usually available. Therefore it is important to investigate SFST adaptive control that can handle actuator failures in the presence of model mismatch.

We consider the single reference-input case, (i.e., $r$ is a scalar) with multiple redundant similar actuators. In this case, the columns of the $B_{0}$ matrix are parallel to the reference model input matrix $b_{m} \in R^{n}$, i.e., the $i$ th column of $B_{0}$ is:

$$
b_{i}=b_{m} / \alpha_{i}, i=1, \ldots, m
$$

for some unknown $\alpha_{i}$ whose sign is assumed to be known.

The adaptive control input is:

$$
v=\widehat{K}_{1}^{T} x+\widehat{k}_{2} r+\widehat{k}_{3}
$$

where $\widehat{K}_{1} \in R^{m \times n}$ and $v(t), \widehat{k}_{2}, \widehat{k}_{3} \in R^{m}$. It is assumed that at least one actuator remains functional.

\section{A. Model Mismatch in System Matrix Only}

We shall first consider the case where the system's " $B$ " matrix is known, i.e., $\delta b=0$, and model mismatch occurs only in the " $A$ " matrix. In this case, the closed-loop plant becomes

$$
\begin{aligned}
\dot{x}= & \left(A_{0}+\delta a\right) x+B_{0}(I-\sigma) v+B_{0} \sigma \bar{u} \\
= & \left(A_{0}+\delta a+B_{0}(I-\sigma) K_{1}^{T}\right) x+B_{0}(I-\sigma) \widetilde{K}_{1}^{T} x+B_{0}(I-\sigma) K_{2} r \\
& +B_{0}(I-\sigma) \widetilde{K}_{2} r+B_{0}(I-\sigma) k_{3}+B_{0}(I-\sigma) \widetilde{k}_{3}+B_{0} \sigma \bar{u}
\end{aligned}
$$

where $\widetilde{K}_{1}=\widehat{K}_{1}-K_{1}$ etc. There can be up to $m-1$ actuator failures. We assume that the following matching conditions are satisfied for the nominal plant $\left(A_{0}, B_{0}\right)$ for some $K_{1}, k_{2}$, and $k_{3}$, and for all actuator failure patterns $(\sigma)$.

$$
A_{0}+B_{0}(I-\sigma) K_{1}=A_{m} ; B_{0}(I-\sigma) K_{2}=b_{m} ; B_{0}(I-\sigma) k_{3}=B_{0} \sigma \bar{u}
$$

Therefore (41) can be written as

$$
\dot{x}=\left(A_{m}+\delta a\right) x+b_{m} r+B_{0}(I-\sigma)\left(\widetilde{K}_{1}^{T} x+\widetilde{k_{2}} r+\widetilde{k_{3}}\right)
$$


To accommodate the plant-model mismatch, the following perturbed reference model is employed:

$$
\dot{x}_{m}=\left(A_{m}+\widehat{\delta} a\right) x_{m}+b_{m} r
$$

where $\widehat{\delta} a$ denotes the estimate of $\delta a$. Thus the error equation is

$$
\dot{e}=A_{M} e-\widetilde{\delta} a x+B_{0}(I-\sigma)\left(\widetilde{K}_{1}^{T} x+\widetilde{k_{2}} r+\widetilde{k}_{3}\right)
$$

where $A_{M}=A_{m}+\widehat{\delta a}$. The following theorem presents the adaptation and parameter estimation laws that ensure that $e(t) \rightarrow 0$ and all signals remain bounded. As in Section IIB, we shall first assume for simplicity of presentation that $\widehat{\delta a}$ remains within the quadratic stability limits. This will be ensured by employing parameter projection as in section IIC.

Theorem 2 For the system given by (41), (44), the control law (40) with gain adaptation laws

$$
\begin{aligned}
\hat{\widehat{K_{1 j}}} & =-\operatorname{sgn}\left(\alpha_{j}\right) \Gamma_{1 j} x e^{T} P b_{m} \\
\dot{\hat{k_{2 j}}} & =-\operatorname{sgn}\left(\alpha_{j}\right) \gamma_{2 j} b_{m}^{T} P e r \\
\dot{\hat{k_{3 j}}} & =-\operatorname{sgn}\left(\alpha_{j}\right) \gamma_{3 j} b_{m}^{T} P e
\end{aligned}
$$

for $j=1, \ldots, m$ and parameter estimation law

$$
\dot{\widehat{\delta a}}=\Gamma_{a} P e x^{T}
$$

where $\Gamma_{1 j} \in R^{n \times n}, \Gamma_{a} \in R^{n \times n}$ are positive definite and symmetric, and $\gamma_{2 j}, \gamma_{3 j}$ are positive scalars, guarantee that all closed-loop signals including adaptive gains and parameter estimates are bounded and the tracking error $e(t)=x(t)-x_{m}(t)$ goes to zero as $t \rightarrow \infty$.

Outline of proof- Eq. (45) can be written as

$$
\begin{aligned}
\dot{e} & =A_{M} e-\widetilde{\delta} a x+\sum_{j \notin \mathcal{J}_{p}}^{m} b_{j}\left(\widetilde{K}_{1 j}^{T} x+\widetilde{k}_{2 j} r+\widetilde{k}_{3 j}\right) \\
& =A_{M} e-\widetilde{\delta} a x+b_{m} \sum_{j \notin \mathcal{J}_{p}}^{m}\left(1 / \alpha_{j}\right)\left(\widetilde{K}_{1 j}^{T} x+\widetilde{k_{2 j}} r+\widetilde{k}_{3 j}\right)
\end{aligned}
$$

where $\mathcal{J}_{p}=j_{1}, j_{2}, \ldots, j_{p}$ denotes the set of indices corresponding to the $p \leq m-1$ failed actuators. Similar to $[2]$, define

$$
V=e^{T} P e+\sum_{j \notin \mathcal{J}_{p}}^{m} \frac{1}{\left|\alpha_{j}\right|}\left(\widetilde{K}_{1 j}^{T} \Gamma_{1 j}^{-1} \widetilde{K}_{1 j}+\widetilde{k}_{2 j}^{2} \gamma_{2 j}^{-1}+\widetilde{k}_{3 j}^{2} \gamma_{3 j}^{-1}\right)+\sum_{i=1}^{n} \widetilde{\delta} a_{i}{ }^{T} \Gamma_{a}^{-1} \widetilde{\delta} a_{i}
$$

Then

$$
\begin{aligned}
& \dot{V}=2 e^{T} P\left[A_{M} e-\widetilde{\delta a x}+b_{m}\left\{\sum_{j \notin \mathcal{J}_{p}}^{m}\left(\widetilde{K}_{1 j}^{T} x+\widetilde{k_{2 j}} r+\widetilde{k}_{3 j}\right) / \alpha_{j}\right\}\right] \\
& +2 \sum_{j \notin \mathcal{J}_{p}}^{m} \frac{1}{\left|\alpha_{j}\right|}\left({\widetilde{K_{1 j}}}^{T} \Gamma_{1 j}^{-1} \widehat{\widehat{K}}_{1 j}+\widetilde{k}_{2 j} \gamma_{2 j}^{-1} \widehat{k}_{2 j}^{\cdot}+\widetilde{k}_{3 j} \gamma_{3 j}^{-1} \widehat{k}_{2 j}\right)+\sum_{i=1}^{n} \widetilde{\delta} a_{i}{ }^{T} \Gamma_{a}^{-1} \dot{\widehat{\delta}} a_{i} \\
& =-2 e^{T} Q e-2 \operatorname{Tr}\left[\widetilde{\delta a}^{T}\left(P e x^{T}-\Gamma_{a}^{-1} \dot{\hat{\delta a}}\right)\right]+2 \sum_{j \notin \mathcal{J}_{p}}^{m}\left(\frac{{\widetilde{K_{1 j}}}^{T} x e^{T} b_{m}}{\alpha_{j}}+\frac{{\widetilde{K_{1 j}}}^{T} \Gamma_{1 j}^{-1} \dot{\overline{K_{1 j}}}}{\left|\alpha_{j}\right|}\right) \\
& +2 \sum_{j \notin \mathcal{J}_{p}}^{m}\left(\frac{\widetilde{k_{2 j}} e^{T} b_{m} r}{\alpha_{j}}+\frac{\widetilde{k_{2 j}} \gamma_{2 j}^{-1} \widehat{\hat{k_{2 j}}}}{\left|\alpha_{j}\right|}\right)
\end{aligned}
$$

Setting the update equations as in the theorem statement, we get

$$
\dot{V} \leq-e^{T} Q e
$$


The remainder of the proof is similar to that of Theorem 1 and the results in Ref [2].

Remark- To ensure that $\widehat{\delta a}$ remains within the quadratic stability bounds, parameter projection similar to Section IIC needs to be employed.

The adaptive scheme implementation and model updates are performed as outlined in Section IID.

The more general case with unmatchable uncertainties in both $A$ and $B$ is considered next.

\section{B. Model Mismatch in Both System- and Input- Matrices}

When model mismatch occurs in the input matrix (in addition to the system matrix), it is not possible to completely mitigate the effect of stuck actuators. However, it is possible to estimate the plant-model mismatch in the system- and input- matrices as well as the uncompensated bias term.

The system equation is:

$$
\begin{aligned}
\dot{x} & =\left(A_{0}+\delta a\right) x+\left(B_{0}+\delta b\right) u \\
& =\left(A_{0}+\delta a\right) x+\left(B_{0}+\delta b\right)(I-\sigma) v+\left(B_{0}+\delta b\right) \sigma \bar{u}
\end{aligned}
$$

where

$$
u={\widehat{K_{1}}}^{T} x+\widehat{k_{2}} r+\widehat{k_{3}}=K_{1}^{T} x+k_{2} r+k_{3}+\left({\widetilde{K_{1}}}^{T} x+\widetilde{k_{2}} r+\widetilde{k_{3}}\right)
$$

Again we assume that the matching conditions (42) hold for the nominal plant $\left(A_{0}, B_{0}\right)$ for up to $m-1$ failures. Denoting

$$
\begin{aligned}
\delta b_{u} & =\delta b(I-\sigma) \\
\xi & =\delta_{b} \bar{u}
\end{aligned}
$$

(which are piecewise constant) and using the matching conditions (42), (54) can be written as:

$$
\dot{x}=\left(A_{m}+\delta a+\delta b_{u} \widehat{K}_{1}^{T}\right) x+\left(b_{m}+\delta b_{u} \widehat{k}_{2}\right) r+B_{0}(I-\sigma)\left[\widetilde{K}_{1}^{T} x+\widetilde{k}_{2} r+\widetilde{k}_{3}\right]+\xi+\delta b_{u} \widehat{k}_{3}
$$

This plant cannot match the reference model dynamics (2). To accommodate the plant-model mismatch and the non-compensable bias input terms (the last two terms in Eq. 57), we modify the reference model as:

$$
\dot{x}_{m}=A_{M} x_{m}+b_{M} r+\widehat{\xi}+\widehat{\delta} b_{u} \widehat{k}_{3}
$$

where

$$
\begin{aligned}
A_{M} & =A_{m}+\widehat{\delta} a+\widehat{\delta} b_{u} \widehat{K}_{1}^{T} \\
b_{M} & =b_{m}+\widehat{\delta} b_{u} \widehat{k}_{2}
\end{aligned}
$$

and $\hat{\xi}, \widehat{\delta b}_{u}$ are estimates of $\xi, \delta b_{u}$. From (54) and (58), we have

$$
\begin{aligned}
\dot{e}= & A_{M} e-\widetilde{\delta} a x-\widetilde{\delta} b_{u}\left(\widehat{K}_{1}^{T} x+\widehat{k}_{2} r+\widehat{k}_{3}\right) \\
& +B(I-\sigma)\left[\widetilde{K}_{1}^{T} x+\widetilde{k}_{2} r+\widetilde{k}_{3}\right]+\widetilde{\xi}-\widetilde{\delta} b_{u} \widehat{k}_{3}
\end{aligned}
$$

where $\tilde{\xi}=\hat{\xi}-\xi ; \widetilde{\delta b}_{u}=\widehat{\delta b}_{u}-\delta b_{u}$.

The following theorem presents gain adaptation and parameter estimation laws that ensure that all signals remain bounded and that $e(t) \rightarrow 0$ as $t \rightarrow \infty$. Note, however, that the modified reference model (58), (59) has a bias term and may not represent the ideal closed-loop dynamics, even if $A_{M}, b_{M}$ are satisfactory based on quadratic stability and/or $H_{2} / H_{\infty}$ performance criteria. This is due to non-compensable mismatch resulting from input bias, which no control law can eliminate.

As in Section IIIA, we shall first assume for simplicity of presentation that $\widehat{\delta A}$ remains within the quadratic stability limits. This will be ensured by employing parameter projection as in section IIC.

Theorem 3 For the system given by (54), (58), the control law (55) with gain adaptation laws

$$
\begin{aligned}
\dot{\widehat{K_{1 j}}} & =-\operatorname{sgn}\left(\alpha_{j}\right) \Gamma_{1 j} x e^{T} P b_{m} \\
\dot{\hat{k_{2 j}}} & =-\operatorname{sgn}\left(\alpha_{j}\right) \gamma_{2 j} b_{m}^{T} P e r \\
\dot{\hat{k_{3 j}}} & =-\operatorname{sgn}\left(\alpha_{j}\right) \gamma_{3 j} b_{m}^{T} P e
\end{aligned}
$$


for $j=1, \ldots, m$ and parameter estimation laws

$$
\begin{aligned}
\dot{\hat{\delta a}} & =\Gamma_{a} P e x^{T} \\
\dot{\delta b_{u}} & =\Gamma_{b} P e v^{T} \\
\dot{\hat{\xi}} & =-\Gamma_{\xi} P e
\end{aligned}
$$

where $\Gamma_{1 j} \in R^{n \times n}, \Gamma_{a} \in R^{n \times n} \Gamma_{b} \in R^{n \times n}, \Gamma_{\xi} \in R^{n \times n}$ are positive definite and symmetric, and $\gamma_{2 j}, \gamma_{3 j}$ are positive scalars, guarantee that all closed-loop signals including adaptive gains and parameter estimates are bounded and the tracking error $e(t)=x(t)-x_{m}(t)$ goes to zero as $t \rightarrow \infty$.

Outline of proof- Eq. (60) can be written as

$$
\begin{aligned}
\dot{e} & =A_{M} e-\widetilde{\delta a} x-\widetilde{\delta} b_{u} v+\sum_{j \notin \mathcal{J}_{p}}^{m} b_{j}\left(\widetilde{K}_{1 j}^{T} x+\widetilde{k}_{2 j} r+\widetilde{k}_{3 j}\right)+\tilde{\xi} \\
& =A_{M} e-\widetilde{\delta} a x-\widetilde{\delta} b_{u} v+b_{m} \sum_{j \notin \mathcal{J}_{p}}^{m}\left(1 / \alpha_{j}\right)\left(\widetilde{K}_{1 j}^{T} x+\widetilde{k_{2 j}} r+\widetilde{k}_{3 j}\right)+\tilde{\xi}
\end{aligned}
$$

where $\mathcal{J}_{p}=j_{1}, j_{2}, \ldots, j_{p}$ denotes the set of indices corresponding to the $p \leq m-1$ failed actuators. Similar to [2], define

$$
\begin{aligned}
V= & e^{T} P e+\sum_{j \notin \mathcal{J}_{p}}^{m} \frac{1}{\left|\alpha_{j}\right|}\left(\widetilde{K}_{1 j}^{T} \Gamma_{1 j}^{-1} \widetilde{K}_{1 j}+\widetilde{k}_{2 j}^{2} \gamma_{2 j}^{-1}+\widetilde{k}_{3 j}^{2} \gamma_{3 j}^{-1}\right)+\sum_{i=1}^{n} \widetilde{\delta} a_{i}{ }^{T} \Gamma_{a}^{-1} \widetilde{\delta} a_{i} \\
& +\sum_{i=1}^{m} \widetilde{\delta b}_{u i}^{T} \Gamma_{b}^{-1} \widetilde{\delta b}_{u i}+\tilde{\xi}^{T} \Gamma_{\xi}^{-1} \tilde{\xi}
\end{aligned}
$$

Then

$$
\begin{aligned}
& \dot{V}=2 e^{T} P\left[A_{M} e-\widetilde{\delta a x}-\widetilde{\delta} b_{u} v+b_{m}\left\{\sum_{j \notin \mathcal{J}_{p}}^{m}\left(\widetilde{K}_{1 j}^{T} x+\widetilde{k_{2 j}} r+\widetilde{k}_{3 j}\right) / \alpha_{j}\right\}+\tilde{\xi}\right] \\
& +2 \sum_{i=1}^{n} \widetilde{\delta} a_{i}^{T} \Gamma_{a}^{-1} \dot{\hat{\delta}} a_{i}+2 \sum_{i=1}^{m} \widetilde{\delta} b_{u i}^{T} \Gamma_{b}^{-1} \dot{\hat{\delta}} b_{u i}+2 \tilde{\xi}^{T} \Gamma_{\xi}^{-1} \dot{\widehat{\xi}} \\
& =-2 e^{T} Q e-2 \operatorname{Tr}\left[\widetilde{\delta a}^{T}\left(P e x^{T}-\Gamma_{a}^{-1} \dot{\widehat{\delta a}}\right)\right]-2 \operatorname{Tr}\left[\widetilde{\delta b}_{v}^{T}\left(P e u^{T}-\Gamma_{b}^{-1} \dot{\hat{\delta b}}\right)\right]+2 \widetilde{\xi}^{T}\left(P e+\Gamma_{\xi}^{-1} \dot{\widehat{\xi}}\right) \\
& +2 \sum_{j \notin \mathcal{J}_{p}}^{m}\left(\frac{\widetilde{K_{1 j}}{ }^{T} x e^{T} b_{m}}{\alpha_{j}}+\frac{\widetilde{K_{1 j}}{ }^{T} \Gamma_{1 j}^{-1} \hat{\widehat{K_{1 j}}}}{\left|\alpha_{j}\right|}\right)+2 \sum_{j \notin \mathcal{J}_{p}}^{m}\left(\frac{\widetilde{k_{2 j}} e^{T} b_{m} r}{\alpha_{j}}+\frac{\widetilde{k_{2 j}} \gamma_{2 j}^{-1} \hat{\hat{k}_{2 j}}}{\left|\alpha_{j}\right|}\right)
\end{aligned}
$$

Setting the update equations as in the theorem statement, we get

$$
\dot{V} \leq-e^{T} Q e
$$

The remainder of the proof is similar to that of Theorem 2.

Remark- To ensure that $\widehat{\delta A}$ remains within the quadratic stability bounds, parameter projection similar to Section IIC needs to be employed.

As stated previously, although $x(t) \rightarrow x_{m}(t)$ as $t \rightarrow \infty$, the reference model state vector $x_{m}(t)$ may not be satisfactory (even if $A_{M}, b_{M}$ are acceptable) because of the non-compensable bias term $\left(\widehat{\xi}+\widehat{\delta b}_{u} \widehat{k}_{3}\right)$ in (58). If the bias term is small, the reference model may be acceptable.

The adaptive scheme implementation and model updates are performed in a manner similar to Section IID. 


\section{Example}

This section illustrates the algorithm introduced in the paper via an example. The nominal model $\left(A_{0}\right.$, $\left.B_{0}\right)$ is

$$
A_{0}=\left[\begin{array}{cc}
0 & 1 \\
-1.5 & 1
\end{array}\right] ; B_{0}=\left[\begin{array}{l}
0 \\
1
\end{array}\right] \text {. }
$$

The unstable nominal model is stabilized by $K_{1}=[-3,-3]^{T}$, and the reference model is defined as the closed-loop system:

$$
A_{m}=A_{0}+B_{0} K_{1}^{T}=\left[\begin{array}{cc}
0 & 1 \\
-4.5 & -2
\end{array}\right], B_{m}=\left[\begin{array}{l}
0 \\
1
\end{array}\right] \text {. }
$$

Suppose the actual system matrix is $A_{0}+\delta a$, where $\delta a=\left[\begin{array}{cc}0.2 & 0.2 \\ 0 & 0\end{array}\right]$, and the input matrix is $B_{0}$ (that is, $\delta b=0)$. Since $\delta b=0$ we need to estimate only $\delta a, K_{1}$, and $K_{2}$. Clearly the matrix $A_{0}+\delta a+B_{0} K_{1}^{T}$ will not have the same structure as $A_{m}$ for any $K_{1}$. The algorithm, introduced in previous sections, allows the reference model system matrix $A_{m}+\widehat{\delta a}+\widehat{\delta b} \widehat{K}_{1}^{T}$ in Eq. (9) to accommodate the plant-model mismatch, and the states of the system to track those of the reference model.

The first step is to compute the permissible limits on $\widehat{\delta A}$ for quadratic stability of the time-varying reference system. This can be accomplished using an LMI formulation as descried in section IIA. The maximum permissible variation $\widehat{\Delta A}=\widehat{\delta a}$ in Eq. (10) was obtained as $\widehat{\delta a_{11}}$ and $\widehat{\delta_{12}} \in[-0.5465,0.5465]$. The parameter projection described in Sec IIC was not implemented in this example, and the permissible variation threshold was arbitrarily set at 80 percent of the maximum permissible limits, i.e., [-0.4372, 0.4372]. The corresponding Lyapunov matrix $P$ in Eq. (14) (with $Q=0$, i.e., stability was the only requirement) was obtained as

$$
P=\left[\begin{array}{ll}
608.8390 & 167.7162 \\
167.7162 & 132.6987
\end{array}\right]
$$

The gains $\Gamma_{1}, \Gamma_{2}, \Gamma_{a}$, and $\Gamma_{b}$ in Eqs. (17)and(18) were selected as 20, 20, [0.3 $0 ; 0$ 0.1], and [1 $0 ; 0$ 1], respectively.

A reference input $r(t)$ consisting of a noisy square wave superimposed on a step function was applied (Figure 1). Figure 2 shows the tracking performance without adaptation. The tracking errors are on the order of 0.1 .

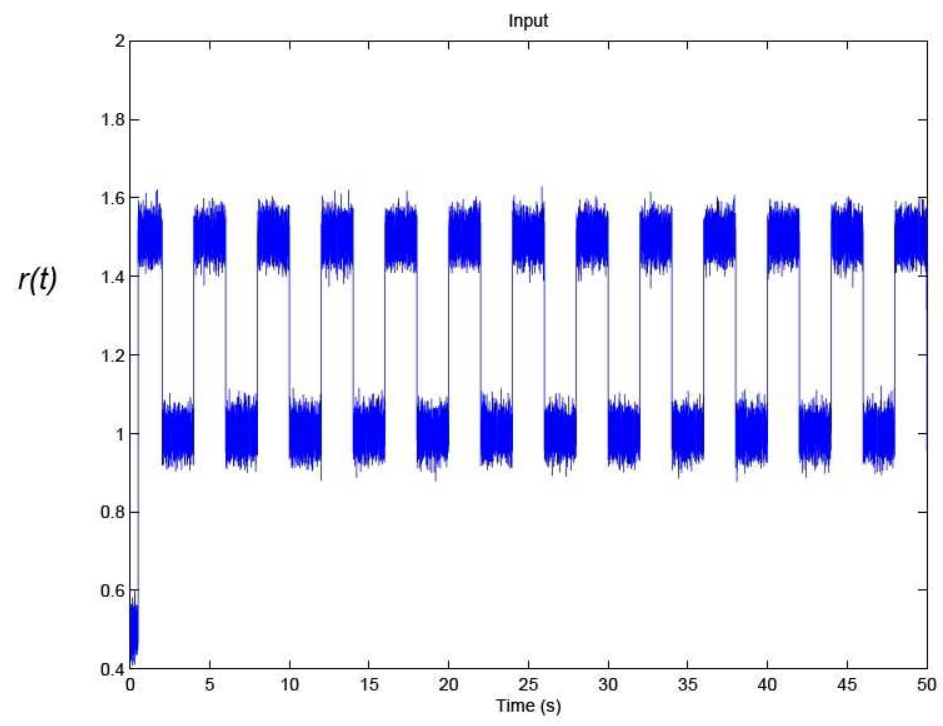

Figure 1. The time history of the reference input $r(t)$ 


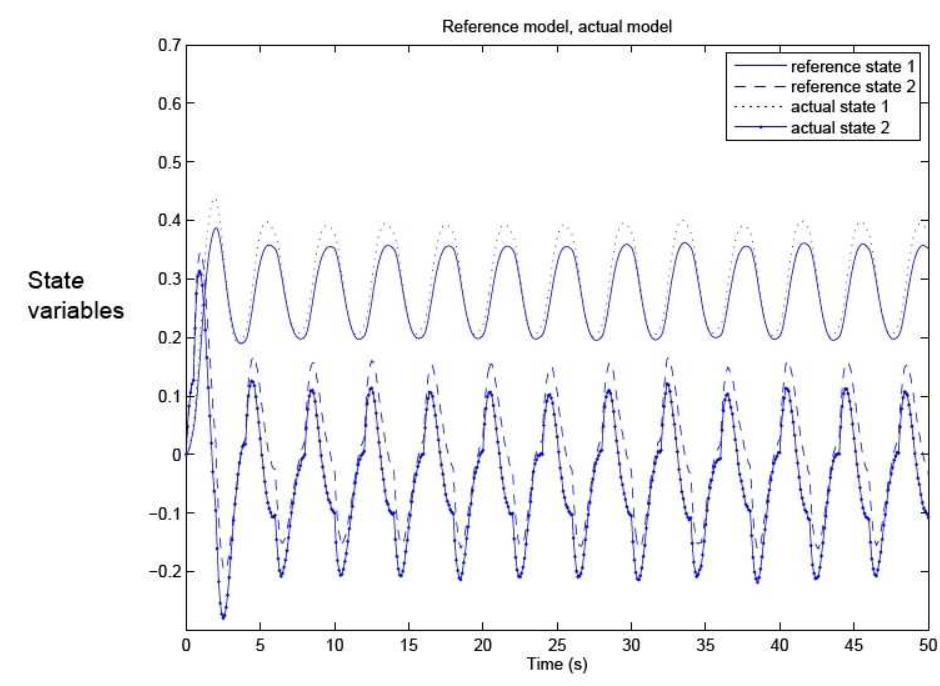

(a) Plant and reference model states

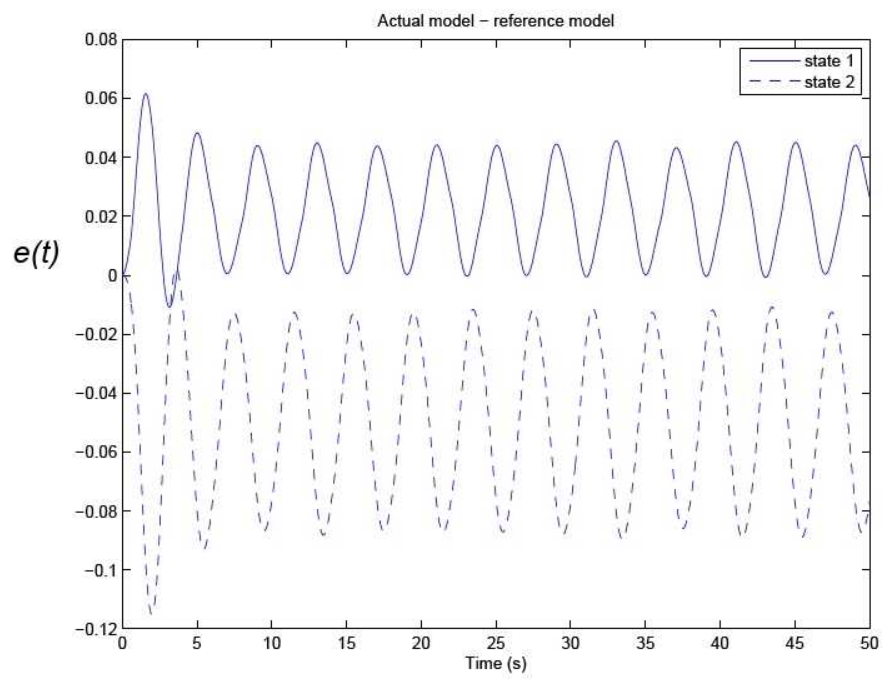

(b) State tracking error

Figure 2. Tracking performance with no adaptation $\left(\delta a=\left[\begin{array}{lll}0.2 & 0.2 ; & 0\end{array}\right]\right)$

Next, the simulation was performed with the adaptive control law. Figure 3 shows the tracking performance with adaptation. It can be seen that the tracking errors are much smaller (by an order of magnitude) and remain $<10^{-3}$ for $t \geq 20$. Figure 4 shows the parameter estimates $\widehat{\delta a_{11}}$ and $\widehat{\delta a_{12}}$. It can be seen that

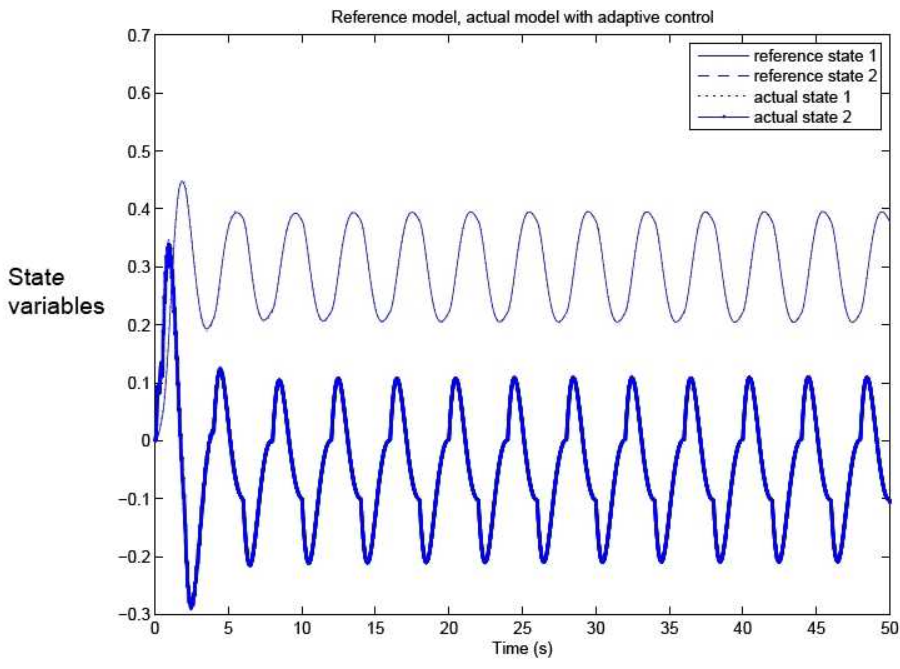

(a) Plant and reference model states
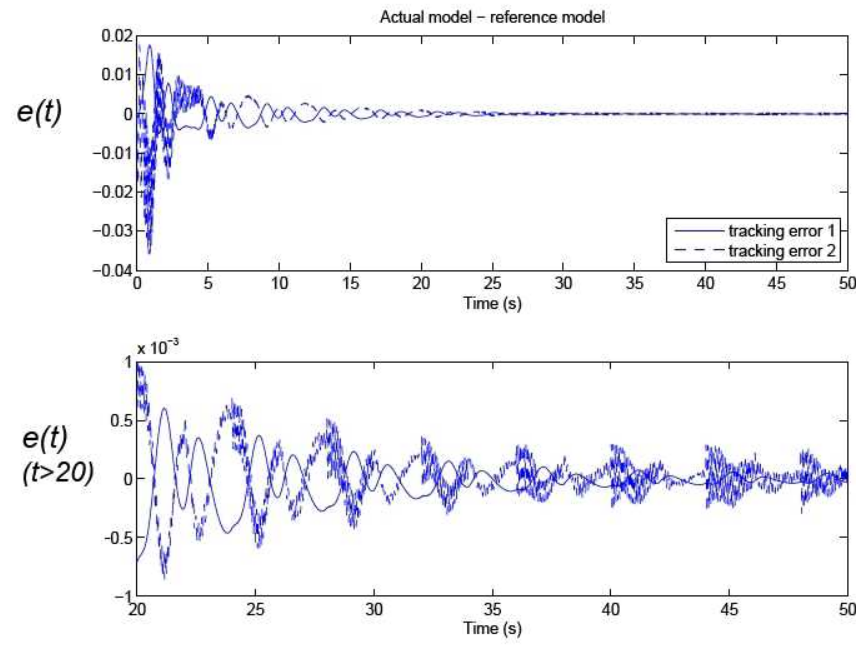

(b) State tracking error

Figure 3. Tracking performance with adaptation $\left(\delta a=\left[\begin{array}{llll}0.2 & 0.2 ; & 0 & 0\end{array}\right)\right.$

the estimates approach within 5 per cent of the true parameter values in less than 25 seconds. Since the parameter estimates remained within the permissible limits, it was not necessary to update/redesign the reference model. However, at $t=25 \mathrm{sec}$, it would be desirable to redesign the reference model based on the updated nominal model, in order to obtain a better response. 


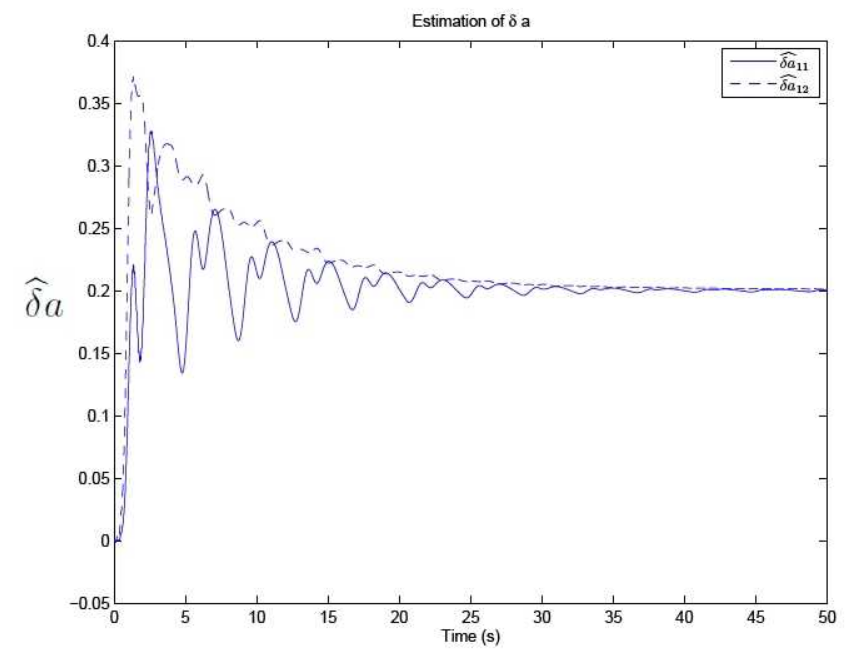

Figure 4. Estimates of $\delta a_{11}$ and $\delta a_{12}$ for $\delta a=\left[\begin{array}{lll}0.2 & 0.2 ; 0 & 0\end{array}\right]$

The mismatch matrix was next increased to: $\delta a=\left[\begin{array}{cc}0.2 & 0.4 \\ 0 & 0\end{array}\right]$. Figure 5 shows the parameter estimates, which approach the true values, but at a slower rate (within 10 per cent in about $100 \mathrm{sec}$ ). The state tracking error (not shown), however, approaches zero much faster., similar to the previous case. Again, the estimates $\widehat{\delta a}_{11}$ and $\widehat{\delta a}_{12}$ remained within the permissible bounds, hence reference model redesign/update was not necessary, although such an update would enhance the system performance.

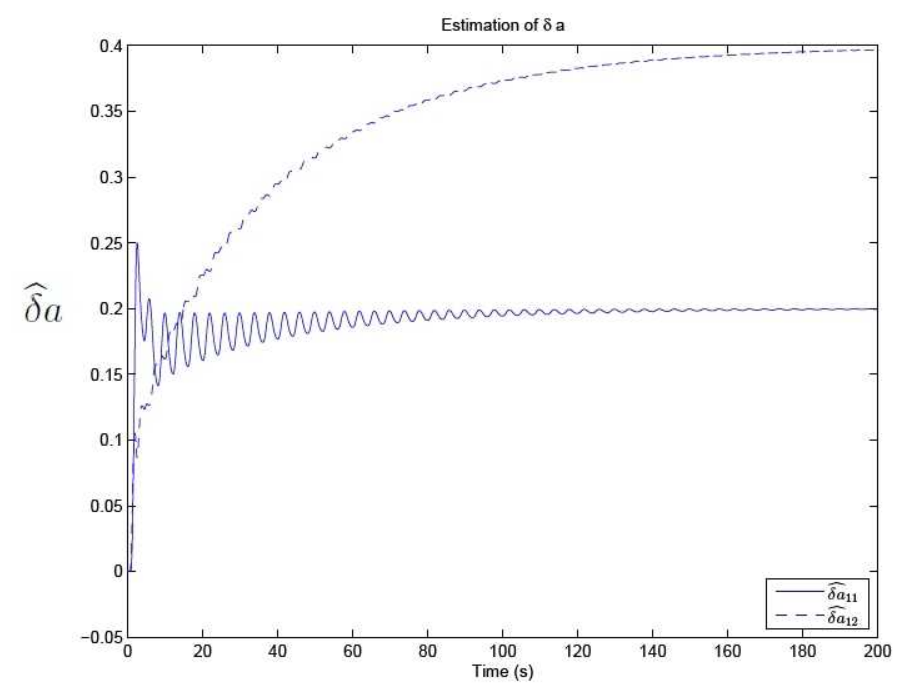

Figure 5. Estimates of $\delta a_{11}$ and $\delta a_{12}$ for $\delta a=[0.20 .4 ; 00]$

The mismatch matrix was next increased further to $\delta a=\left[\begin{array}{cc}0.6 & 0.5 \\ 0 & 0\end{array}\right]$. In this case, the parameter estimate $\widehat{\delta a_{11}}$ exceeded the threshold which was set at \pm 0.4372 ( 80 per cent of maximum permissible values), at approximately $5 \mathrm{sec}$ and the reference model was redesigned using $\widehat{A_{0}}($ new $)=\widehat{A_{0}}+\widehat{\delta a}$, with $\widehat{K_{1}}, \widehat{K_{2}}$ reset at new values that accomplish plant-model matching. The new permissible bounds on $\widehat{\delta a_{11}}, \widehat{\delta a_{11}}$ were calculated. These bounds were exceeded at approximately $12.5 \mathrm{sec}$, when the reference model was once again redesigned. The reference input was a chirp signal, which was found to be more effective than the step and square wave used previously, probably because of the larger magnitude of the mismatch. Figure 6 shows 
the system states and the reference model-states as well as the state tracking errors, with no adaptation, and Figure 7 shows the results with adaptation. The state tracking performance with adaptive control is very good.

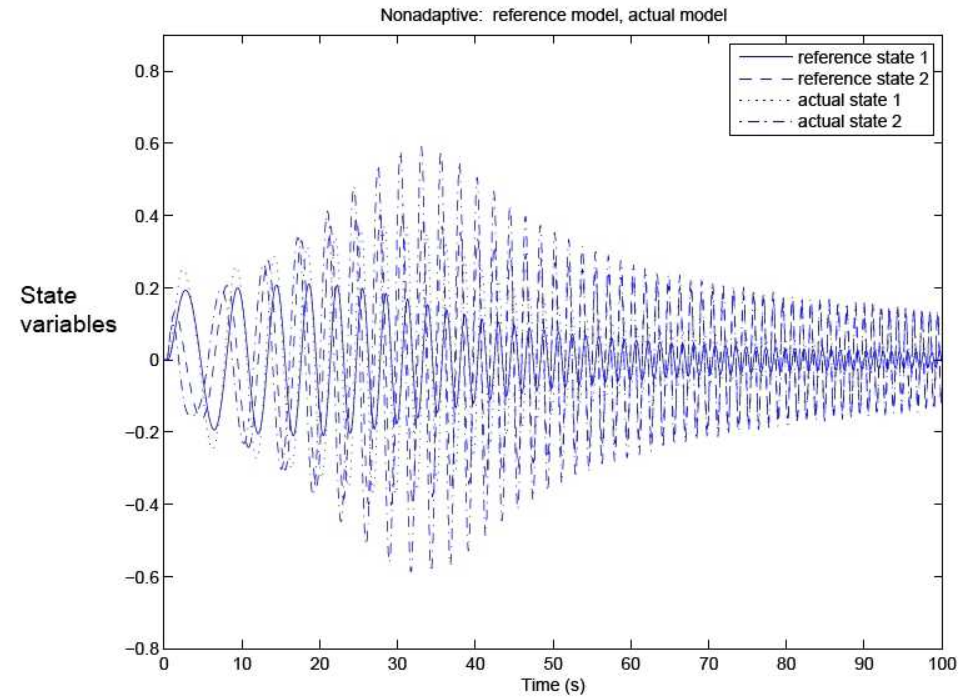

(a) Plant and reference model states

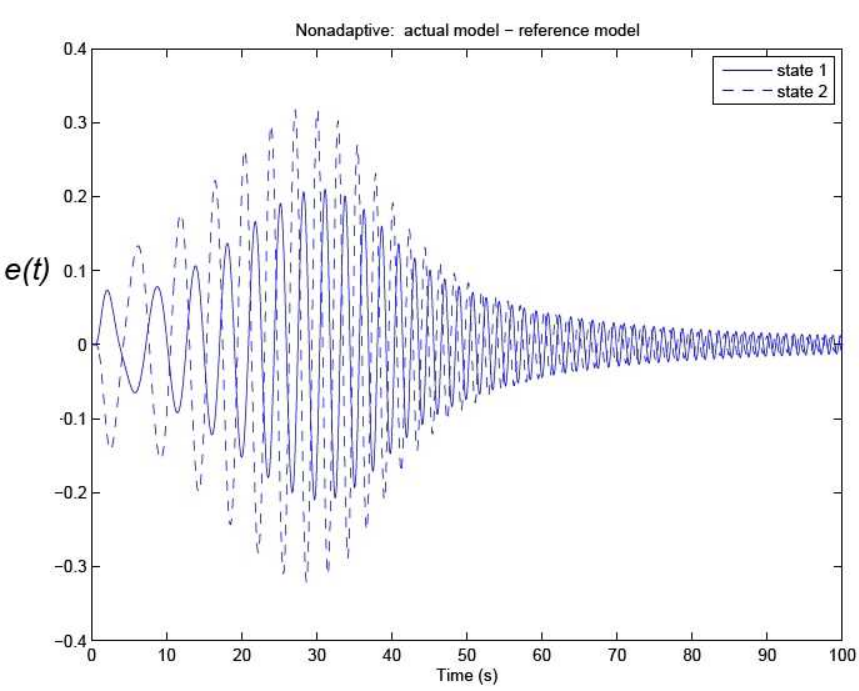

(b) State tracking error

Figure 6. Tracking performance with no adaptation $\left(\delta a=\left[\begin{array}{llll}0.6 & 0.5 ; & 0 & 0\end{array}\right]\right)$

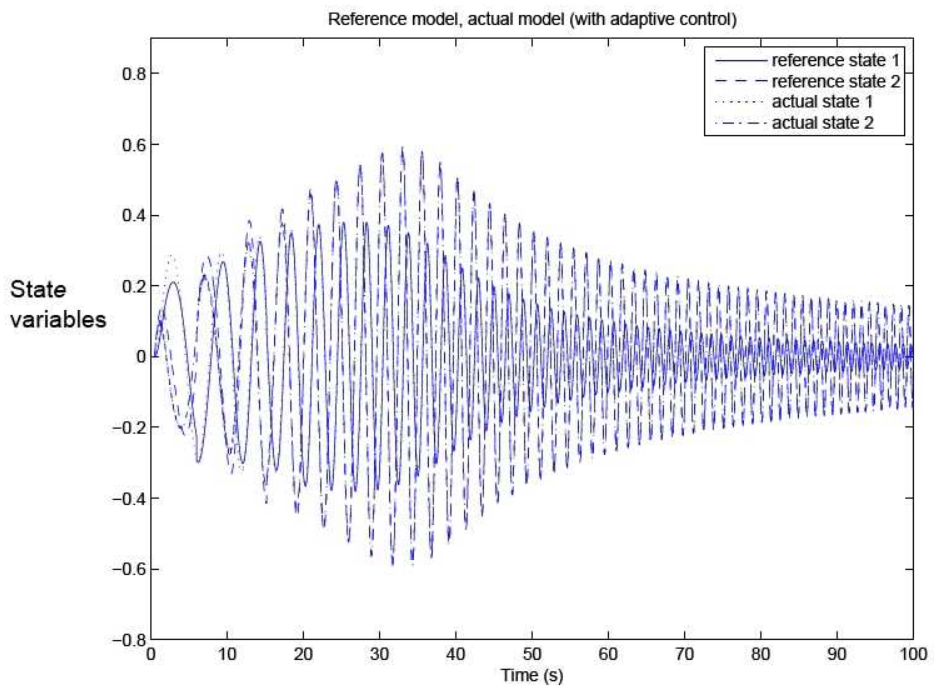

(a) Plant and reference model states

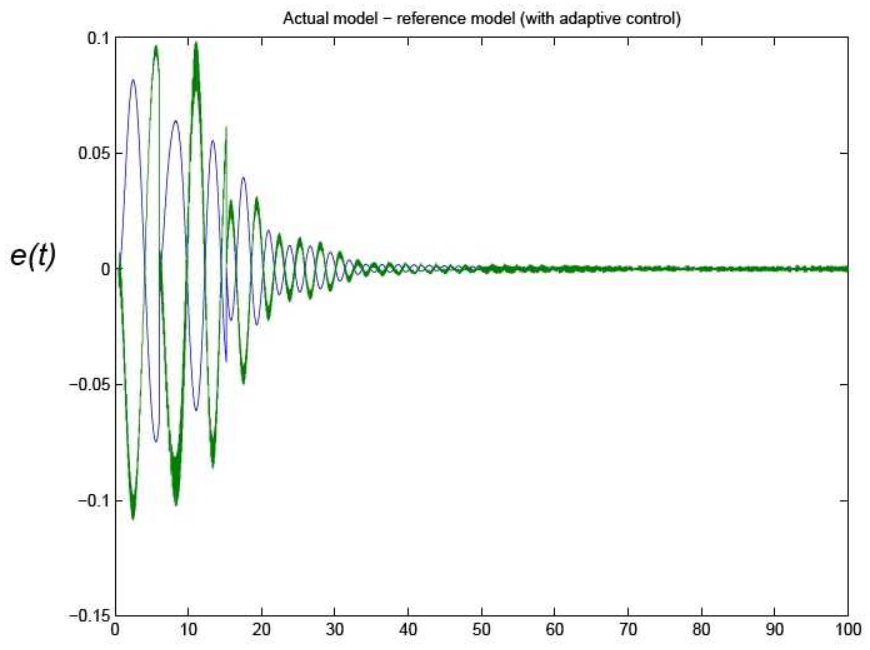

(b) State tracking error

Figure 7. Tracking performance with adaptation $\left(\delta a=\left[\begin{array}{llll}0.6 & 0.5 ; & 0 & 0\end{array}\right]\right)$

As seen in Figure 8, the parameter estimates appear to approach their true values (relative to the most recent plant estimate) in about 50 sec.

\section{Discussion}

The focus of the paper has been on theoretical aspects of how the plant-model mismatch can be estimated (in the presence or absence of actuator failures) and how the reference model can be changed to accommodate the mismatch while ensuring stability and asymptotic tracking. From the limited numerical 


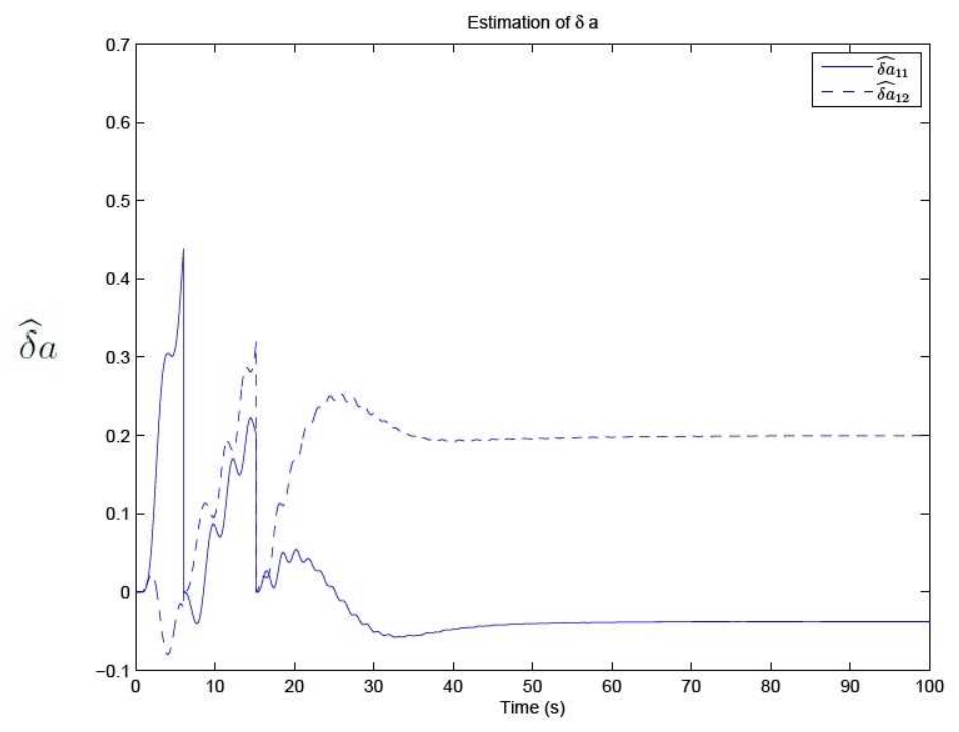

Figure 8. Estimation of $\delta a_{11}$ and $\delta a_{12}$ for $\delta a=\left[\begin{array}{llll}0.6 & 0.5 ; & 0 & 0\end{array}\right]$

results obtained, the simultaneous mismatch estimation and gain adaptation can work well for moderate magnitudes of mismatch. The state tracking error approaches zero fairly quickly, although the mismatch estimates approach their steady state values at a slower rate. The mismatch estimates can exhibit a large overshoot initially, which could trigger a redesign of the reference model (because of exceeding the permissible bounds). The parameter estimates approach their true values; however, based on several simulation runs, the type, the magnitude, and the frequency content of the persistent excitation signal are important for accomplishing convergence of the mismatch estimates to their true values. If the persistent excitation signal is not sufficiently rich, the mismatch estimates may approach some constants that are not their true values. Design of appropriate persistent excitation signals, that enable rapid and accurate mismatch estimation without causing unacceptable disturbance in the system, remains an area of future research. In all cases, the state tracking performance was very good and the tracking error quickly approached zero. Further simulation studies are continuing for more realistic systems in order to assess the method and to investigate improvements.

It would be desirable to avoid large over/undershoots in the mismatch parameter estimates, possibly by using techniques such as sigma modification. Also, the performance of the adaptive scheme depends significantly on the choice of the adaptation weighting parameters; therefore it would be of interest to investigate systematic methods for selecting these parameters.

One issue that needs to be addressed is parametrization of the plant-model mismatch. It is straightforward to uniquely parametrize the mismatch when the nominal system is in a canonical form. However, for general system structures, the mismatch $\delta a, \delta b$ would be fully populated matrices. Such a mismatch structure would result in overparametrization. Therefore it would be desirable to investigate canonical structures of plant-model mismatch.

The method used to ensure stability of the reference model was based on quadratic stability, which is usually conservative. In particular, the number of (simultaneous) quadratic stability LMIs grows exponentially with the number of mismatch parameters, which can result in overly conservative bounds on the mismatch. In addition, the LMI solution and reference model redesign are performed in real time, which can be computationally challenging.

Finally, it is desirable to investigate application of the method to realistic higher order examples with several mismatch parameters, and in the presence of actuator failures as described in Section III.

\section{Concluding Remarks}

Direct adaptive control for state tracking using state feedback has been known to be a promising technique for control of uncertain systems. However, it requires rather stringent matching conditions on the plant and 
the reference model which may be violated due to modeling errors and plant changes such as dynamics changes caused by damage and icing in aircraft. To address this issue, this paper presented a new adaptive control approach for state tracking with simultaneous plant-model mismatch estimation that provides a method for changing the reference model when the plant changes are large. In particular, when the estimated plantmodel mismatch exceeds pre-determined bounds, the reference model is redesigned so that the matching conditions are re-established and the overall performance is satisfactory. The method was also extended to the case with actuator failures. Results of application to an example problem indicate that accurate mismatch estimation and satisfactory tracking performance can be obtained in the presence of plant-model mismatch. Further investigation is in progress to evaluate and refine the method.

\section{Acknowledgement}

The authors are grateful to Prof. Carsten Scherer of Delft University of Technology for his help with LMI implementation.

\section{References}

${ }^{1}$ G. Tao and S. M. Joshi: "Direct Adaptive Control of Systems with Actuator Failures: State of the Art and Continuing Challenges", Proc. 2008 AIAA Guidance, Navigation, and Control Conference, Honolulu, HI, Aug 18-21, 2008. 2004.

${ }^{2}$ G. Tao, S. H. Chen, X. D. Tang and S. M. Joshi, "Adaptive Control of Systems with Actuator Failures", Springer, March

${ }^{3}$ G. Tao, S. M. Joshi, and X. Ma: "Adaptive State Feedback and Tracking Control of Systems with Actuator Failures", IEEE Trans. on Automatic Control, vol. 46, No. 1, January 2001, pp. 78-95.

${ }^{4}$ T. E. Gibson, L. G. Crespo, A. M. Annaswamy: "Adaptive Control of Hypersonic Vehicles in the Presence of Modeling Uncertainties", Proc. 2009 American Control Conference, St. Louis, MO, June 10-12, 2009.

${ }^{5}$ C. Cao and N. Hovakimyan: "L1 Adaptive Output Feedback Controller for Non Strictly Positive Real Reference Systems with Applications to Aerospace Examples",Proc. 2008 AIAA Guidance, Navigation, and Control Conference, Honolulu, HI, Aug 18-21, 2008.

${ }^{6}$ G. Tao, Adaptive Control Design and Analysis, N.J.: John Wiley \& Sons, 2003.

${ }^{7}$ A. M. Annaswamy, J. Jang, and E. Lavretsky: "Adaptive gain-scheduled controller in the presence of actuator anomalies", Proc. 2008 AIAA Guidance, Navigation, and Control Conference, Honolulu, HI, Aug 18-21, 2008. 